\title{
Panel on Green and Sustainable IS
}

\author{
Barbara Pernici \\ Politecnico di Milano, piazza Leonardo da Vinci 32, 20133 Milano, Italy \\ barbara.pernici@polimi.it
}

\begin{abstract}
The panel on Green and Sustainable Information Systems has the goal of providing a forum for discussing research issues on this topic within the Information Systems Engineering research community. Information systems, for their pervasive nature in most of human activities that have an IT support, can give a contribution to improve the environmental impact of IT in two main directions: on one side, information systems can provide a support to improve awareness and to control energy efficiency in a variety of areas, such as for instance smart cities and smart buildings, traffic control, and utility management, on the other hand, information systems themselves use computing resources and related facilities in data center and offices, and therefore they have an impact on the environment. While a great emphasis has been given to produce hardware equipment that is energy efficient, only recently the theme of considering energy efficiency in connection to information systems and their design has emerged. The panel will discuss the different types of green and sustainable information systems and will discuss emerging research topics and possible future research developments and goals.
\end{abstract}

